\title{
Frequency Dependence of Electrical Coupling in Deiters" Cells of the Guinea Pig Cochlea
}

\author{
Laura Lagostena, Andres Cicuttin, Juan Inda, Bechara Kachar \& Fabio \\ Mammano
}

To cite this article: Laura Lagostena, Andres Cicuttin, Juan Inda, Bechara Kachar \& Fabio Mammano (2001) Frequency Dependence of Electrical Coupling in Deiters" Cells of the Guinea Pig Cochlea, Cell Communication \& Adhesion, 8:4-6, 393-399, DOI: 10.3109/15419060109080760

To link to this article: https://doi.org/10.3109/15419060109080760

曲 Published online: 11 Jul 2009.

Submit your article to this journal $₫$

Џll Article views: 186

Citing articles: 2 View citing articles 


\title{
Frequency Dependence of Electrical Coupling in Deiters' Cells of the Guinea Pig Cochlea
}

\author{
LAURA LAGOSTENA \\ Biophysics Sector and INFM Unit, International School for Advanced Studies, via Beirut 2-4, 34014 Trieste, Italy \\ ANDRES CICUTTIN \\ Mircroprocessor Laboratory ICTP/INFN, Abdus Salam International Centre for Theoretical Physics, \\ via Beirut 31, 34014 Trieste, Italy \\ JUAN INDA and BECHARA KACHAR \\ Section on Structural Cell Biology. NIDCD. NIH. Bethesda MD. USA \\ FABIO MAMMANO \\ Biophysics Sector and INFM Unit, International School for Advanced Studies, via Beirut 2-4. 34014 Trieste. Italy
}

\begin{abstract}
Immunolabeling with antibodies against connexins 26 and 30 showed that, in the guinea pig cochlea, supporting Deiters' cells are massively interconnected and form an orderly network within the organ of Corti. In paired patch-clamp recordings the coupling ratio (CR) of adjacent Deiters' cells at the apex of the cochlea $(\sim 0.31)$ was 3 -fold smaller than in isolated cell pairs due to shunting afforded by multicellular connectivity. With sinusoidal current stimuli the delay in signal propagation between adjacent cells increased with increasing frequency whereas the amplitude did not change significantly up to $200 \mathrm{~Hz}$ (corner frequency $F_{c} \sim 220 \mathrm{~Hz}$ ). Depolarizing voltage commands applied to an outer hair cell ( $\mathrm{OHC}$ ) elicited outward potassium currents in the OHC and inward currents in the abutting Deiters' cells, supplying direct evidence for potassium buffering in the organ of Corti. Computational analysis indicates that electrical signals injected into a Deiters' cell are transmitted across a network segment spanning 8 cell diameters. Thus electrical coupling in the organ of Corti is unlikely to influence the selectivity of frequency filtering performed mechanically by the mammalian cochlea.
\end{abstract}

Keywords Caged compounds, cell networks, connexins, electrical models, hearing, organ of Corti, patch clamp, UV photolysis

\section{INTRODUCTION}

In mammals, theperception and frequency discrimination of sound is due to the fine organization and strict co-operation between sensory and supporting cells of the auditory epithelium, the organ of Corti of the cochlea. One particular type of supporting cells, the Deiters' cells, join mechanically the

Received 1 September 2001; Accepted 15 September 2001.

Supported by: grant Progetto di Ricerca Avanzata CADY, Istituto Nazionale di Fisica della Materia; grant N.9906194385 (Cofinanziamento), Ministero dell'Universita e della Ricerca Scientifica (to F.M.); intramural research project Z01 DC 00002-11 from the National Institutes on Deafness and other Communication Disorders (to B.K.). We thank: Renato Nobili for helpful discussions and for the drawing reproduced in Figure 1A; Elisa Margotti for help with immunohistochemistry.

Address correspondence to Dr. Fabio Mammano, International School for Advanced Studies, via Beirut 2-4, 34014 Trieste, Italy. E-mail: mammano@sissa.it 


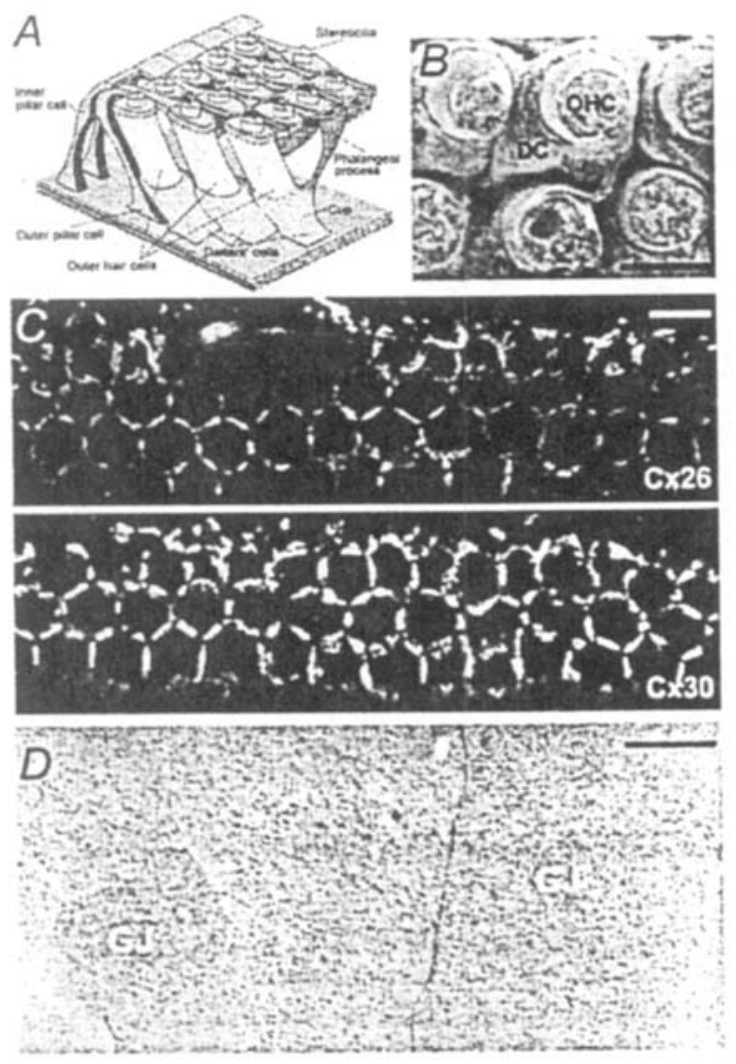

FIG. 1. Cellular architecture of Deiters' cells in the organ of Corti and patterns of gap junction interconnectivity. $A$, schematic representation of the central portion of the organ of Corti. Note the strict contact between adjacent Deiters' cells at the level of their cup (basolateral) region. $B$, scanning electron microscopy view of an organ of Corti segment: OHC, outer hair cells; DC, supporting Deiters' cells. $C$, immunofluorescence confocal optical section showing the distribution of connexin $26(\mathrm{Cx} 26)$ and $30(\mathrm{Cx} 30)$ between adjacent Deiters' cells in a whole mount preparation of the organ of Corti. $D$, freeze-fracture replica of the plasma membranes of a Deiters' cell showing large gap junction patches (GJ). Scale bars: B,C, $10 \mu \mathrm{m} ; \mathrm{D}$, $0.5 \mu \mathrm{m}$.

OHCs to the basilar membrane (Figure 1A), forming an integral part of the feed-back loop that subtends the "cochlear amplifier" (Nobili et al. 1998). Soundinduced oscillations of the organ of Corti modulate the opening probability of hair cell transduction channels generating receptor potentials (Dallos et al. 1982). Oscillatory waveforms similar to receptor potentials, but with 5 to 10 -fold attenuated amplitude, have been recorded from supporting cells in vivo (Oesterle and Dallos 1989; 1990). Gap junctions are known to couple all supporting cells in the organ of Corti (Santos-Sacchi 2000) and have been implicated in the passage of electrical signals (Oesterle and Dallos 1990). However, the extent of the coupling, its frequency dependence and modulation have not been determined. Here we have used a range biophysical and microscopy techniques to investigate electrical coupling in the guinea-pig organ of Corti. Experimental results have been compared to computer simulations performed on a distributed network model of supporting cells coupled by gap junctions.

\section{MATERIALS AND METHODS}

The preparation and methods of recording from sensory (Mammano et al. 1995) and supporting Deiters' cells (Lagostena and Mammano 2001) in the intact organ of Corti have been described previously. In addition, fluorescence imaging of intracellular $\mathrm{Ca}^{2+}$ and $\mathrm{UV}$ flash photolysis were also performed as described by Lagostena and Mammano (2001). Immunofluorescence was performed as described in Mammano et al. (1999) using antibodies against connexin 26 and 30 , and FITC-conjugated secondary antibodies (Zymed, San Francisco, CA). For freeze-fracture and thin section microscopy, specimens were also prepared as described in Mammano et al. (1999). For computer simulations, the response of a model network of Deiters' cells to injection of current at selected nodes (Figure 4A) was computed with the use of circuit simulator Spice (University of California at Berkeley). Amplitude and frequency of the stimuli were selected to match experimental values. Results are expressed as mean \pm S.E.M., $n=$ number of cells (or cell couples, for paired recordings).

\section{RESULTS AND DISCUSSION}

\section{Gap Junction Distribution in Deiters' Cells}

Deiters' cells refiect the organization of the OHCs forming a compact staggered pattern (scanning electron micrograph in Figure 1B). Immunofluorescence 
labeling for connexin 26 and 30 , the predominant connexin isoforms in the organ of Corti (Forge et al. 1999; Kikuchi et al. 1995; Lautermann et al. 1998), showed three rows of Deiters' cells interconnected by gap junctions to form a tight planar hexagonal network (Figure 1C). Freeze-fracture along plasma membranes of Deiters' cell showed multiple large patches of gap junctions (Figure 1D).

\section{Patch-Clamp Recordings from Deiters' Cells in the Organ of Corti}

In the presence of extracellular octanol ( $1 \mathrm{mM})$, a well known uncoupling agent effective on gap junctions in the organ of Corti (Mammano et al. 1996), depolarizing steps applied to Deiters' cells elicited outward currents that were blocked by $20 \mathrm{mM}$ TEA, indicating that they were due to activation of $\mathrm{K}^{+}$-selective channels as previously reported (Nenov et al. 1998). Hyperpolarizing steps elicited negligible voltage-dependent responses, resulting in a strong outward rectification of the I-V curve. The zero-current potential of these pharmacologically isolated cells was $V_{z}=-21.4 \pm 2.7 \mathrm{mV}(\mathrm{n}=5)$. $\mathrm{V}_{\mathrm{z}}$ shifted in the depolarized direction to $\mathrm{V}_{\text {TEA }}=$ $-5.5 \pm 1.5 \mathrm{mV}(\mathrm{n}=3)$ in the presence of TEA $(20 \mathrm{mM})$. The input conductance, i.e. the conductance around $\mathrm{V}_{\mathrm{z}}$, decreased from $2.0 \pm 0.7 \mathrm{nS}$ $(n=5)$ to $0.8 \pm 0.2 \mathrm{nS}(\mathrm{n}=3)$, indicating that a fraction of the TEA-sensitive current was activated near $V_{z}$. Positive to $V_{z}$ the slope conductance reached a maximum of $17.5 \pm 2.6 \mathrm{nS}$ at a potential $V_{\max }=27.7 \pm 1.5 \mathrm{mV}(\mathrm{n}=5)$. Cell capacitance, measured by the capacitance compensation circuitry of the amplifier, was $34.6 \pm 2.3 \mathrm{pF}(n=5)$. To investigate electrical coupling between adjacent Deiters' cells connected to the network, we used paired recordings in the current-clamp mode (Figure 2A) while bathing the organ of Corti in normal (i.e., octanol free) extracellular solution. Bias currents were adjusted to keep the pre-stimulus potential of both cells (conventionally defined as 1 and 2) near $0 \mathrm{mV}$, where the cell input conductance was $15.5 \pm 2.4 \mathrm{nS}(\mathrm{n}=5)$. Application of current steps

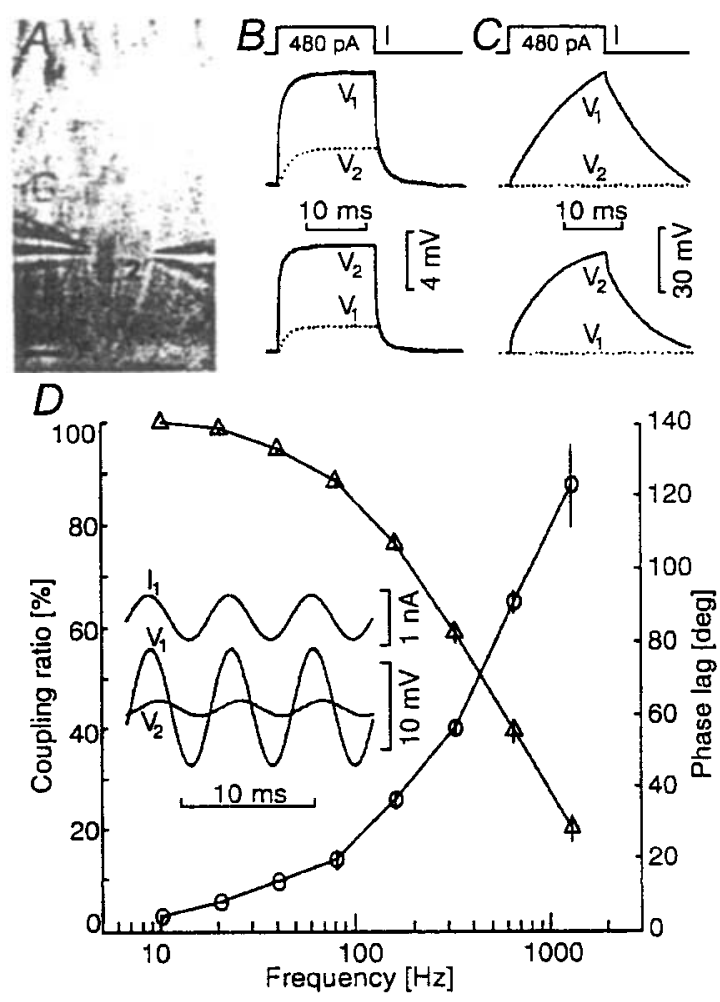

FIG. 2. Measurement of coupling ratio in pairs of Deiters' cells within the organ of Corti. $\boldsymbol{A}$, video image showing a patch-clamped pair of adjacent cells ( 1 and 2) in the third row of Deiters' cells. Scale bar: $10 \mu \mathrm{m} . B$, top: current waveform $(I=480 \mathrm{pA})$ injected alternatively into cell $I$ and cell 2 ; middle, voltage responses evoked in cell $1\left(\mathrm{~V}_{1}\right.$, solid line $)$ and cell $2\left(\mathrm{~V}_{2}\right.$, dotted line $)$ by current injection in 1 ; bottom, responses evoked by exchanging the role of 1 and 2 . $C$, octanol (1 mM) blocks electrical coupling; top: injected current step $(\mathrm{I}=480 \mathrm{pA})$; middle, responses of cell $1\left(\mathrm{~V}_{1}\right.$, solid line $)$ and cell $2\left(\mathrm{~V}_{2}\right.$, dotted line $)$ to current injection in 1 . Bottom: responses obtained by exchanging the role of 1 and 2 ( $B$ and $C$ are from the same cell pair). $D$, frequency dependence of sine waves transmitted through gap junctions. Inset, membrane voltage oscillations $\left(\mathrm{V}_{1}\right.$ and $\left.V_{2}\right)$ produced by injecting sinusoidal current $\left(I_{1}=960\right.$ pA peak-topeak, $163 \mathrm{~Hz}$ ) in cell 1. Triangles (left axis): coupling ratio shown as percentage of the value taken at the lowest frequency, $10.2 \mathrm{~Hz}$; circles (right axis), phase lag of $V_{2}$ relative to $V_{1}$ (in degrees). Each point is the mean \pm S.E.M. (vertical bars through symbols) of $n=3$ recordings from different pairs. The cut-off frequency $\left(F_{c}=219 \mathrm{~Hz}\right)$ was determined by linear interpolation between the points at $163 \mathrm{~Hz}$ and $326 \mathrm{~Hz}$.

$(\mathrm{I}=480 \mathrm{pA}, 20 \mathrm{~ms}$; Figure $2 \mathrm{~B}$, top $)$ to cell 1 evoked voltage responses $V_{1}$ and $V_{2}$ that, at stimulus onset, were fitted by saturating double-exponential functions. Time constants were $\tau_{1,1}=0.12 \pm 0.02 \mathrm{~ms}$, $\tau_{2.1}=1.7 \pm 0.2 \mathrm{~ms}$ for $\mathrm{V}_{1} ; \tau_{1.2}=0.52 \pm 0.13 \mathrm{~ms}$, 
$\tau_{2.2}=2.6 \pm 0.5 \mathrm{~ms}$ for $\mathrm{V}_{2}(\mathrm{n}=8)$. The amplitude of $V_{1}$ measured in the steady state was $6.0 \pm 0.5 \mathrm{mV}$ $(n=11)$ whereas $C R \equiv V_{2} / V_{1}$ was $0.31 \pm 0.03$ $(n=11)$. Voltage responses were also evoked in both cells when current was injected in cell 2 , indicating bi-directionality of the coupling (Figure 2B, middle and bottom). Octanol (1 $\mathrm{mM})$ completely suppressed the adjacent cell's response (Figure 2C). Furthermore, the longer of the two time constants (i.e. $\tau_{2,1}$ and $\tau_{2.2}$ ) and the magnitude of the voltage response of the injected cell increased considerably, as expected from the increased input resistance following gap junction closure. In all cell pairs tested, the effect of octanol was reversible within $\sim 10$ min after washout. In a few experiments, isolated pairs of Deiters' cells were mechanically obtained by applying gentle suction to neighboring cells with a pipette, yielding $C R$ values in the range $0.660-0.914(n=4)$. The simplest explanation for the significantly smaller mean value of $C R$ in the undisrupted network is that it reflected shunting due to multicellular connectivity seen with microscopy (Figure IC,D). To test if signal transmission through adjacent Deiters' cells in the network was frequencydependent, we injected sinusoidal current stimuli of constant amplitude $\left(I_{1}=960\right.$ pA peak-to peak $)$, ranging in frequency between 10.2 and $1305 \mathrm{~Hz}$. As shown in Figure 2D, the magnitude of CR (left axis) decreased whereas the phase lag of $V_{2}$ relative to $V_{1}$ (right axis) increased monotonically with increasing frequency. The mean corner frequency $F_{c}$, i.e. the frequency at which $\mathrm{CR}$ equaled $(1 / 2)^{1 / 2}$ of its zero-frequency limit, was $219 \pm 20 \mathrm{~Hz}(\mathrm{n}=3)$. Above $1 \mathrm{kHz}$, the lag accumulated by $\mathrm{V}_{2}$ relative to $V_{1}$ was in excess of $100 \mathrm{deg}$ and reached $122 \pm$ $22 \mathrm{deg}$ at $1305 \mathrm{~Hz}(\mathrm{n}=3)$, the highest frequency at which phase relationships could be determined reliably.

\section{Effect of UV Photolysis of Caged InsP $\mathbf{P}_{3}$ on Electrical Coupling}

To test the dependence of electrical coupling on the intracellular free $\mathrm{Ca}^{2+}$ concentration $\left(\left[\mathrm{Ca}^{2+}\right]_{i}\right.$ ), cell pairs connected to the network were co-loaded through patch pipettes with caged Ins $\mathrm{P}_{3}(16 \mathrm{~m} \mu)$ and Oregon Green 488 BAPTA-1 (OG, $100 \mathrm{~m} \mu$ ). We have recently demonstrated that rapid fluorescence increases of the OG signaled a $\mathrm{Ca}^{2+}$ rise following delivery of $1-\mathrm{ms}$ UV flashes that caused the liberation of the Ins $\mathrm{P}_{3}$ active form within the cytoplasm (Lagostena and Mammano 2001). Here, using the same experimental approach, we investigated the effect of intracellular $\mathrm{Ca}^{2+}$ on $\mathrm{CR}$ and $\mathrm{F}_{\mathrm{c}}$. In response to stimuli that increased $O G$ fluorescence by $21 \pm 3 \%, C R$ decreased by $72 \pm 8 \%$ whereas $F_{c}$ decreased by $22 \pm 4 \%(n=3)$ indicating that the extent and frequency-dependence of electrical communication were highly susceptible to manipulations affecting the $\left[\mathrm{Ca}^{2+}\right]_{i}$.

\section{Electromechanical Coupling Between OHCs and Deiters' Cells}

Sound-induced voltage oscillations in supporting cells of the cochlea have been attributed to hair-cell activity (Oesterle and Dallos 1989; 1990). However, a currently unresolved problem is how electrical signals are transmitted from sensory to supporting cells. Thin section microscopy in Figure 3A shows the synaptic region of an OHC, with efferent terminals, wrapped by the cup of a Deiters' cell. We performed paired patch-clamp recordings from selected couples formed by an $\mathrm{OHC}$ and its supporting Deiters' cell, both maintained at the holding potential $V_{h}=-50 \mathrm{mV}$ under voltage clamp (Figure 3B). When a $100 \mathrm{mV}$ depolarizing step was applied to the $\mathrm{OHC}$ while keeping the potential of the Deiters' cell at $V_{h}$, outward potassium currents (Mammano and Ashmore 1996) and inward currents were recorded from the OHC and the Deiters' cell, respectively. Electrical responses were accompanied by visible movements of the patched cells (Figure $3 C)$. These clearly originated from the active electromotile responses of the $\mathrm{OHC}$ subjected to the potential step which dragged both its supporting Deiters' cell as well as its nearest neighbors, confirming previous findings (Mammano et al. 1995). As both the OHC and the Deiters' cell were anchored by a patch pipette, most of the movement occurred at the 


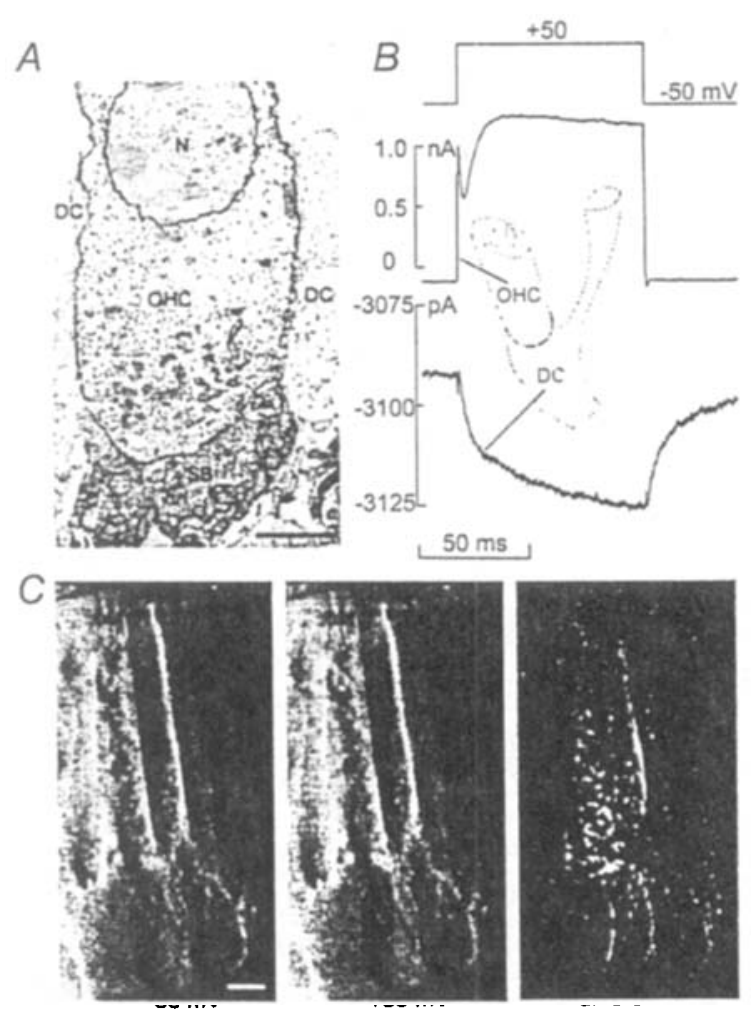

FIG. 3. Electromechanical coupling of $\mathrm{OHC}$ and Deiters' cell probed by paired patch clamp recording in the organ of Corti. $A$, thin section microscopy of a Deiters' cell (DC) cup region tightly wrapped around the basal pole of an outer hair cell $(\mathrm{OHC})$ with efferent synaptic boutons (SB); N, nucleus of the OHC. $B$, top: voltage command imparted to an $\mathrm{OHC}$; middle: current responses recorded simultaneously from the $\mathrm{OHC}$ and $\mathrm{DC}$; holding potential $\mathrm{V}_{\mathrm{h}}=$ $-50 \mathrm{mV}$ for both cells. $C$, left: digital video micrograph of the organ of Corti showing OHCs connected to Deiters' cells; the faint halos of patch pipettes contacting one $\mathrm{OHC}$ and its supporting $\mathrm{DC}$ are also visible; middle: micrograph captured while holding the $\mathrm{OHC}$ to +50 $\mathrm{mV}$; right: image obtained by digital subtraction of the left and middle micrographs. Only moving parts of the tissue stand out of the pixel noise (see associated video). Scale bars: A, $5 \mu \mathrm{m}$; C, $10 \mu \mathrm{m}$.

cell interface (Figure 3C, difference). Similar results were obtained in two other pair recordings. In these experiments, voltage commands could be applied hundreds of times in rapid sequence without appreciable deterioration in either structural or electrical coupling, indicating that the connection at the cell interface was mechanically robust and kept the contact zone tight also under extreme stress. Deiters' cell electrical responses following $\mathrm{OHC}$ stimulation likely originated from potassium influx across the supporting cell membrane abutting the $\mathrm{OHC}$ base, possibly involving the activation of stretch sensitive channels in the supporting cell as previously suggested (Oesterle and Dallos 1989). Lack of gap junction connections between the two cell types (Forge et al. 1999; Kikuchi et al. 1995; Lautermann et al. 1998) permits to exclude direct electrotonic coupling.

\section{Results of Computer Simulations}

We modeled Deiters' cells as a planar hexagonal network (Figure 4A) of identical nodes (cells) joined by identical links (gap junction connections). We assigned fixed values to node elements $R_{M}$ (membrane resistance, $60 \mathrm{M} \Omega$ ) and $\mathrm{C}_{\mathrm{M}}$ (cell capacitance, $30 \mathrm{pF}$ ), reflecting the passive properties of the Deiters' cell membrane. Knowledge of the microscopic parameters needed to determine precise values for the link elements $R_{D}, R_{L}$ and $L$ is lacking. Therefore, to match simultaneously the time-domain and the frequency-domain responses of the network a trial-and-error parameter selection procedure was adopted. Responses to current steps were computed for fixed values of $R_{L}=55 \mathrm{M} \Omega, R_{D}=3 \mathrm{G} \Omega$ and $\mathrm{L}=7$ kHenry while varying the number of diagonal rows, $N_{r}$, from 2 to 8 (Figure 4B). Increasing $N_{r}$ produced a simultaneous decrease of the response time constants and the $\mathrm{CR}$, asymptotically approaching experimental values. Figure $4 \mathrm{C}$ (left) shows the frequency response for $\mathrm{N}_{\mathrm{r}}=2,4$ and 8. Larger numbers of diagonal rows (up to $\mathrm{N}_{\mathrm{r}}=64$ ) did not improve significantly model responses neither in the time- nor in the frequency-domain. Instead, parametric analysis showed that decreasing inductance $L$ while keeping $\mathrm{N}_{\mathrm{r}}=8$ produced minor changes in the magnitude but a drastic deviation of the phase response (data not shown). Finally, introducing a capacitance $C_{j}$ in the link circuit while keeping the junctional resistance constant at its optimal value produced a mismatch between model and data. The discrepancy increased dramatically both in magnitude and in phase with increasing values of $\mathrm{C}_{\mathrm{j}}$ (Figure $4 \mathrm{C}$, right).

In summary, our data indicate that, at the apex of the cochlea, the Deiters' cell network is capable 


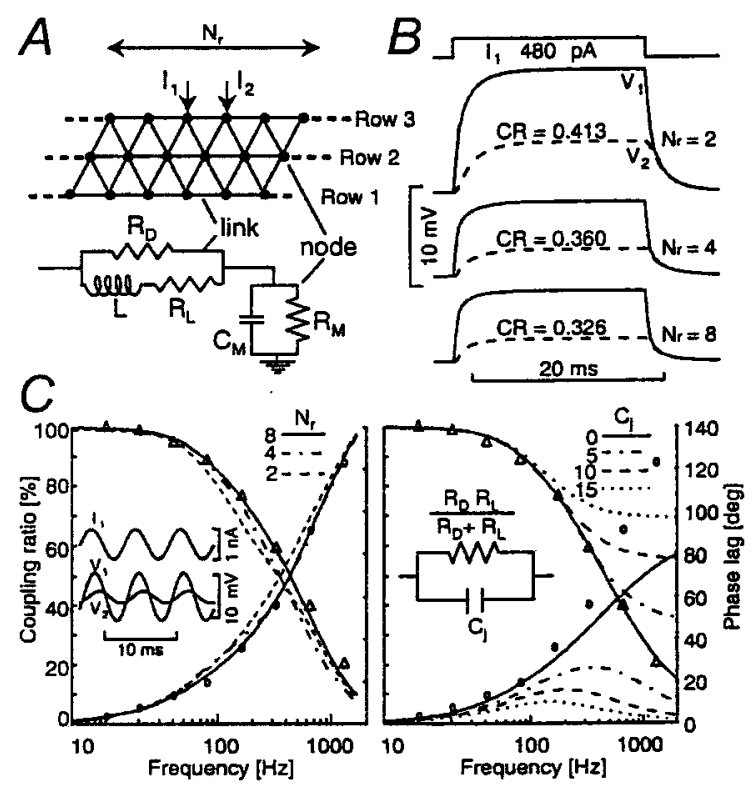

FIG. 4. Electrical model of the network. $A$, top: schematic representation showing three horizontal rows of identical nodes (Deiters' cells, closed symbols) interconnected by identical links (gap junctions, straight segments). $\mathrm{N}_{\mathrm{r}}$ is the number of rows connected by diagonal links (diagonal rows). Downward arrows: current injection points $\left(I_{1}\right.$ and $\left.I_{2}\right)$ in row 3. Bottom: link and node equivalent circuits; $R_{M}=60 \mathrm{M} \Omega$, membrane resistance; $C_{M}=30 \mathrm{pF}$, cell capacitance; $\mathrm{L}=7 \mathrm{kHenry}$, effective inductance. $\mathrm{R}_{\mathrm{L}}=55 \mathrm{M} \Omega, \mathrm{R}_{\mathrm{D}}=$ $3 \mathrm{G} \Omega ; R_{D} \cdot R_{L} /\left(R_{D}+R_{L}\right)$ is junctional resistance. $B$, response to a current step (top), computed for the indicated number of diagonal rows $\mathrm{N}_{\mathrm{r}}$ (bottom); $\mathrm{CR}$, coupling ratio. $C$, simulated frequency dependence of sine waves transmitted through gap junctions. Left axis: coupling ratio $C R=V_{2} / V_{1}$ shown as percentage of the value taken at the lowest frequency $(5 \mathrm{~Hz})$; right axis, phase lag of $\mathrm{V}_{2}$ relative to $\mathrm{V}_{\mathrm{I}}$ (deg). Left graph: solid, dashed, and dash-dot lines: model responses computed for $\mathrm{N}_{\mathrm{r}}=8,4,2$, respectively; circles and triangles: experimental data reproduced from Figure $2 \mathrm{C}$; inset: membrane voltage oscillations $\left(V_{1}\right.$ and $\left.V_{2}\right)$ produced by injecting sinusoidal current $\left(I_{1}=960 \mathrm{pA}\right.$ peak-to-peak, $\left.163 \mathrm{~Hz}\right)$. Right graph: parametric responses computed for increasing values of putative junctional capacitance $\mathrm{C}_{\mathrm{j}}=0$ (solid lines), $5 \mathrm{pF}$ (dash-dot lines), $10 \mathrm{pF}$ (dashed lines) and $15 \mathrm{pF}$ (dotted lines), in parallel with fixed junctional resistance $\mathbf{R}_{\mathbf{D}} \cdot \mathbf{R}_{\mathbf{L}} /\left(\mathbf{R}_{\mathbf{D}}+\mathbf{R}_{\mathbf{L}}\right)$.

of efficiently transmitting electrical signals through gap junctions at frequencies in excess of $220 \mathrm{~Hz}$, a value close to the mechanical resonance frequency of the recording site (Oesterle and Dallos 1989). Results obtained by paired OHC-Deiters' cell recordings suggest that electromotor activity of the OHCs is responsible for the generation of electrical signals in Deiters' cells. Computation results indicate that electrical signals spread longitudinally in a Deiters' cell network segment covering $\sim 8$ cell diameters. In the guinea pig cochlea the peak region of a sharply tuned travelling wave generated by a pure tone spans $\sim 5 \%$ of the basilar membrane length (Mammano and Nobili 1993), or $\sim 100$ cell diameters. Thus, the tonotopic organization of the organ of Corti responses are not smeared by passive propagation of electrical signals through gap junctions. Based on these experiments we conclude that, at least in the apical low-frequency region of the cochlea, Deiters' cell coupling might play a role in the synchronization of cellular responses within the region of maximal mechanical excitation. Finally, it has been proposed that connexin mutations may affect potassium recycling in the organ of Corti (Santos-Sacchi 2000). Our results suggest that, by altering the frequency response of the network, mutations in connexin 26 and 30 (Gasparini et al. 1999) may interfere also with synchronisation of cellular activity and the fine tuning of the mechanical responses to sound input.

\section{REFERENCES}

Dallos, P., Santos-Sacchi, J., and Flock, A. 1982. Intracellular recordings from cochlear outer hair cells. Science 218:582-584.

Forge, A., Becker, D., Casalotti, S. et al. 1999. Gap junctions and connexin expression in the inner ear. Novartis Found Symp 219:134 150.

Gasparini, P., Estivill, X., and Fortina, P. 1999. Vestibular and hearing loss in genetic and metabolic disorders. Curr. Opin. Neurol. 12:3539.

Kikuchi, T., Kimura, R. S., Paul, D. L., and Adams, J. C. 1995. Gap junctions in the rat cochlea: immunohistochemical and ultrastructural analysis. Anat. Embryol. (Berl) 191:101-118.

Lagostena, L., and Mammano, F. 2001. Intracellular calcium dynamics and membrane conductance changes evoked by Deiters' cell purinoceptor activation in the organ of Corti. Cell Calcium 29:191-198.

Lautermann, J., ten Cate, W. J., Altenhoff, P. et al. 1998. Expression of the gap-junction connexins 26 and 30 in the rat cochlea. Cell Tissue Res. 294:415-420.

Mammano, F., and Ashmore, J. F. 1996. Differential expression of outer hair cell potassium currents in the isolated cochlea of the guinea pig. J. Physiol. (Lond) 496 (3):639-646.

Mammano, F., Frolenkov, G., Lagostena, L., et al. 1999. ATP-Induced $\mathrm{Ca}^{2+}$ release in cochlear outer hair cells: localization of an inositol triphosphate-gated $\mathrm{Ca}^{2+}$ store to the base of the sensory hair bundle. J. Neurosci. 19:6918-6929.

Mammano, F., Goodfellow, S. J., and Fountain, E. 1996. Electrophysiological properties of Hensen's cells investigated in situ. Neuroreport 7:537-542.

Mammano, F., Kros, C. J., and Ashmore, J. F. 1995. Patch clamped responses from outer hair cells in the intact adult organ of Corti. Pflugers Arch. 430:745-750. 
Mammano, F., and Nobili, R. 1993. Biophysics of the cochlea: linear approximation. J. Acoust. Soc. Am. 93:3320-3332.

Nenov, A. P., Chen, C., and Bobbin, R. P. 1998. Outward rectifying potassium currents are the dominant voltage activated currents present in Deiters' cells. Hear. Res. 123:168182.

Nobili, R., Mammano, F., and Ashmore, J. F. 1998. How well do we understand the cochlea? Trends Neurosci. 21:159-167.
Oesterie, E. C., and Dallos, P. 1989. Intracellular recordings from supporting cells in the guinea-pig cochlea: AC potentials. $J$ Acoust. Soc. Am. 86:1013-1032.

Oesterle, E. C., and Dallos, P. 1990. Intracellular recordings from supporting cells in the guinea pig cochlea. DC potentials. $J$. Nettrophysiol. 64:617-636.

Santos-Sacchi, J. 2000. Cell coupling in Corti's organ. Brain Res. Brain Res. Rev. 32:167-171. 